\title{
Temperature dependence of Space Charge Limited Current (SCLC) in thin films of silicon nanocrystals
}

\author{
M. A. Rafiq ${ }^{1}$, Y. Tsuchiya ${ }^{2}$, H. Mizuta ${ }^{2}$, Shigeyasu Uno ${ }^{3}$, Z. A. K. Durrani ${ }^{4 *}$ and W. I. Milne ${ }^{4}$
}

${ }^{1}$ Microelectronics Research Centre, Cavendish Laboratory, University of Cambridge, Madingley Road, Cambridge CB3 0HE, U. K.

${ }^{2}$ Department of Physical Electronics, Tokyo Institute of Technology, Tokyo 152-8552, Japan, and SORST JST (Japan Science and Technology).

${ }^{3}$ Hitachi Cambridge Laboratory, Madingley Road, Cambridge CB3 0HE, U. K.

${ }^{4}$ Electronic Devices and Materials Group, Engineering Department, University of Cambridge, Trumpington Street, Cambridge CB2 1PZ, U. K., and SORST JST (Japan Science and Technology)

*Phone: +44 $1223764657 \quad$ FAX: +44 1223332662

E-mail: zakd100@cam.ac.uk

The temperature dependence of the conduction mechanism in thin films of silicon nanocrystals was investigated using $\mathrm{Al} / \mathrm{Si}$ nanocrystal $/ p-\mathrm{Si} / \mathrm{Al}$ diodes. The device (Fig. 1) used a $\sim 300 \mathrm{~nm}$ thick Si nanocrystal film, deposited by plasma decomposition of $\mathrm{SiH}_{4}$ [1] on a $p$-Si substrate (resistivity: $10 \Omega \mathrm{cm}$ ). The nanocrystals were $8 \mathrm{~nm} \pm$ $1 \mathrm{~nm}$ in diameter and undoped, with a $\sim 1.5 \mathrm{~nm}$ thick surface $\mathrm{SiO}_{2}$ layer. Figure 1(b) shows a scanning electron micrograph of our film and Fig. 1(c) shows a transmission electron micrograph of a nanocrystal. In the later image, the oxide is $\sim 3 \mathrm{~nm}$ thick, due to thermal processing before microscopy. The film is non-uniform, with $\sim 60 \%$ nanocrystal coverage per layer, and a number density, $N_{n c} \sim 1.2 \times 10^{18}$ $\mathrm{cm}^{-3}$. The film thickness of $300 \mathrm{~nm}$ avoids pinholes across the film. The diodes were defined using 'mesas', fabricated by electron-beam lithography and reactive ion etching. A $\sim 150$ nm-thick sputtered $\mathrm{SiO}_{2}$ layer, wet-etched over the mesa to expose the nanocrystals, was used to support an $\mathrm{Al}$ top contact. The substrate-contact was also fabricated with Al. The diode area, determined by the top Al-Si nanocrystal contact area, was varied from $35 \mu \mathrm{m} \times 35 \mu \mathrm{m}$ to $200 \mu \mathrm{m} \times$ $200 \mu \mathrm{m}$. The diode current scaled with device area. $\mathrm{Al} / \mathrm{Si}$ nanocrystal $/ n^{+}-\mathrm{Si} / \mathrm{Al}$ and $\mathrm{Au} / \mathrm{Si}$ nanocrystal $/ n^{+}-\mathrm{Si} / \mathrm{Au}$ devices were also fabricated and characterised.

The $I-V$ characteristics of a $35 \mu \mathrm{m} \times 35 \mu \mathrm{m}$ diode, measured from $300 \mathrm{~K}$ to $40 \mathrm{~K}$ using a cryogenic temperature needle prober (BCT-43MDC, Nagase \& Co. Ltd.) and a Hewlett Packard 4156A parameter analyser, are shown in Fig. 2 on a log-log plot. The inset shows the room temperature $I-V$ characteristics of the device from $-8 \mathrm{~V}$ to $4 \mathrm{~V}$ (linear scale). Here, positive bias is applied to the substrate-contact, corresponding to a forward biased substrate. From $300 \mathrm{~K}$ to $200 \mathrm{~K}$, the conduction mechanism is dominated by SCLC transport, in the presence of an exponential distribution of trapping states $[2,8]$. Using this model, we extract a trap density $N_{t}=2.3 \times 10^{17} \mathrm{~cm}^{-3}$ and a characteristic trap temperature $T_{t}=1670 \mathrm{~K}$. Our value of $N_{t}$ is very similar to the nanocrystal number density, $N_{n c} \sim 1.2 \times 10^{18} \mathrm{~cm}^{-3}$. This suggests that only a few carriers are trapped per nanocrystal. In our SCLC current mechanism, holes are injected from the substrate into the nanocrystal film. These carriers can be trapped in the potential well on each nanocrystal. Single-electron or quantum confinement ef- fects can lead to a discrete density of states in the well, limiting the number of carriers trapped in each well. This would explain our observation of similar values of $N_{t}$ and $N_{n c}$. Alternatively, the trapping states may be formed by only a small number of defect states per nanocrystal.

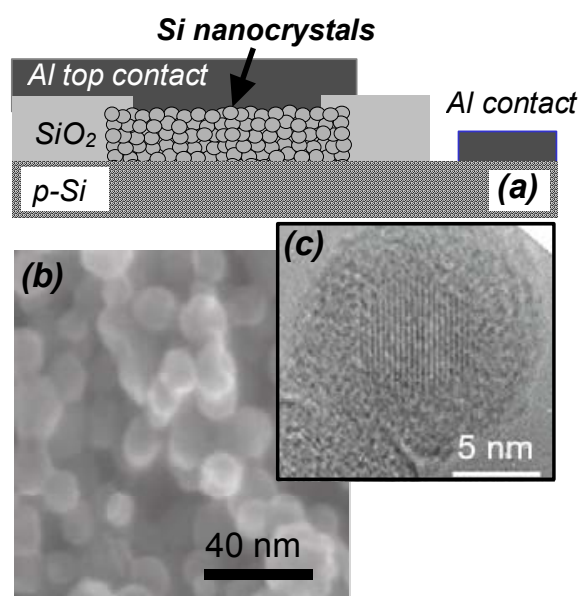

Fig. 1 (a) Schematic of the Al/Si nanocrystal $/ p-\mathrm{Si} / \mathrm{Al}$ diode. (b) Scanning electron micrograph of the $\mathrm{Si}$ nanocrystal film. (c). Transmission electron micrograph of a Si nanocrystal.

The values of $T_{t}=1670 \mathrm{~K}$ (corresponding to $E_{t}=$ $0.14 \mathrm{eV}$ ) and $N_{t}=2.3 \times 10^{17} \mathrm{~cm}^{-3}$ can be compared to amorphous $\mathrm{Si}$ and to other nanocrystal systems. $T_{t}$ does not lie in the range observed for bulk amorphous $\mathrm{Si}(\sim 300 \mathrm{~K}$ to $\sim 1300 \mathrm{~K})$ or for large $(\sim 150 \mathrm{~nm})$ amorphous Si nanoparticles, and $N_{t}$ is two orders of magnitude smaller than in amorphous Si [9]. Our values of $T_{t}$ and $N_{t}$ are more comparable to values observed in CdSe nanocrystals, similar in size to our Si nanocrystals. In CdSe, $T_{t} \sim 1750 \mathrm{~K}, E_{t}=0.15$ $\mathrm{eV}$ and $N_{t} \sim 10^{16}-10^{17} \mathrm{~cm}^{-3}[6,7]$. Our ratio $N_{n c} / N_{t} \approx 5$ is lower than in CdSe nanocrystals, where a ratio of 100 was observed for deep level traps [7]. This suggests more uniform charging in our Si nanocrystals films.

In conclusion, we investigated the conduction 
mechanism in $300 \mathrm{~nm}$-thick Si nanocrystal films, with $\sim 8$ $\mathrm{nm}$ nanocrystals. From $300 \mathrm{~K}$ to $200 \mathrm{~K}$, we observed SCLC hole transport with an exponential distribution of trapping states, where the trap density was $2.3 \times 10^{17} \mathrm{~cm}^{-3}$ and the characteristic trap temperature was $1670 \mathrm{~K}$. The trap density was within an order of magnitude of the nanocrystal number density, suggesting that most nanocrystals trap single or a few carriers at most.

\section{Acknowledgements}

M. A. Rafiq would like to acknowledge the financial support from Cambridge Commonwealth Trust.

\section{References}

[1] S. Oda and M. Otobe. Mater. Res. Soc. Symp. Proc. 358 721 (1995).

[2] M. A. Lampert and P. Mark, Current Injection in Solids (Academic, New York, 1970)

[3] T. A. Burr, A. A. Seraphin, E. Werwa, and K. D. Kolenbrander, Phys. Rev. B 56, 4818 (1997).

[4] M. Ben-Chorin, F. Moller, and F. Koch, Phys. Rev. B 49, 2981 (1994).

[5] C. Peng, K. D. Hirschman, and P. M. Fauchet, J. Appl. Phys. 80, 295 (1996).

[6] D. S. Ginger, and N. C. Greenham, J. Appl. Phys. 87, 1361 (2000).

[7] R. A. M. Hikmet, D. V. Talapin, and H. Weller, J. Appl. Phys. 93, 3509 (2002).

[8] P. Mark, and W. Helfrich, J. Appl. Phys., 33, 205 (1962).

[9] Z. Shen, U. Kortshagen, and S. A. Campbell, J. Appl. Phys., 96, 2204 (2004).

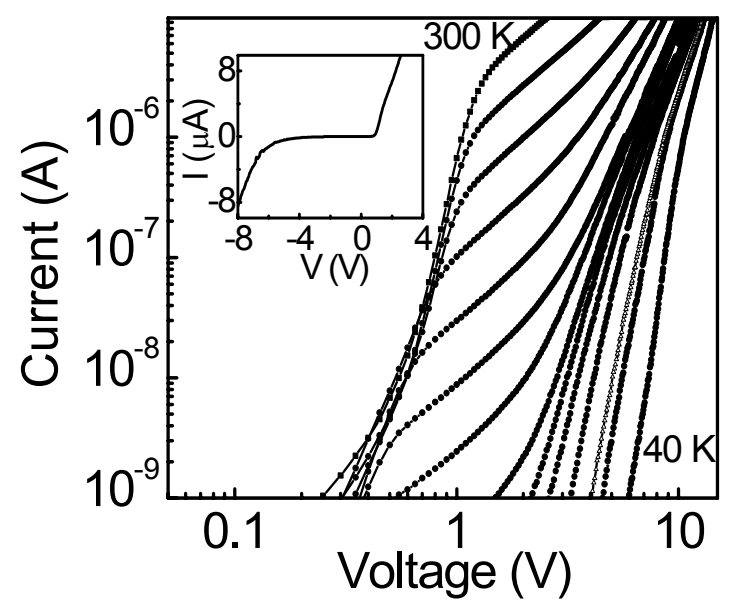

Fig. 2. $I-V$ characteristics of a $35 \mu \mathrm{m} \times 35 \mu \mathrm{m}$ diode from $300 \mathrm{~K}$ to $40 \mathrm{~K}$, on a log-log plot. The temperature step is $20 \mathrm{~K}$. The inset shows the $I-V$ characteristics at $300 \mathrm{~K}$, from $-8 \mathrm{~V}$ to $4 \mathrm{~V}$, on a linear scale. 